hep-th/9905015

May 1999

\title{
Gluino Condensate And Magnetic Monopoles in Supersymmetric Gluodynamics
}

\author{
N. Michael Davies ${ }^{1}$, Timothy J. Hollowood ${ }^{2,3}$, Valentin V. Khoze ${ }^{1}$ \\ And Michael P. Mattis ${ }^{2}$ \\ ${ }^{1}$ Department of Physics, University of Durham, \\ Durham, DH1 3LE, UK \\ n.m.davies@durham.ac.uk, valya.khoze@durham.ac.uk \\ ${ }^{2}$ Theoretical Division T-8, Los Alamos National Laboratory, \\ Los Alamos, NM 87545, USA \\ pyth@schwinger.lanl.gov, mattis@lanl.gov \\ ${ }^{3}$ Department of Physics, University of Wales Swansea, \\ Swansea, SA2 8PP, UK
}

\begin{abstract}
We examine supersymmetric $S U(N)$ gauge theories on $\mathbb{R}^{3} \times S^{1}$ with a circle of circumference $\beta$. These theories interpolate between four-dimensional $\mathcal{N}=1$ pure gauge theory for $\beta=\infty$ and three-dimensional $\mathcal{N}=2$ gauge theory for $\beta=0$. The dominant field configurations of the $\mathbb{R}^{3} \times S^{1} S U(N)$ theories in the semi-classical regime arise from $N$ varieties of monopole. Periodic instanton configurations correspond to mixed configurations of $N$ single monopoles of the $N$ different types. We semi-classically evaluate the non-perturbatively generated superpotential of the $\mathbb{R}^{3} \times S^{1}$ theory and hence determine its vacuum structure. We then calculate monopole contributions to the gluino condensate in these theories and take the decompactification limit $\beta=\infty$. In this way we obtain a value for the gluino condensate in the four-dimensional $\mathcal{N}=1$ supersymmetric $S U(N)$ Yang-Mills theory, which agrees with the previously known 'weak coupling' expression but not with the 'strong coupling' expression derived in the early literature solely from instanton considerations. Moreover, we discover that the superpotential gives a mass to the dual (magnetic) photon, which implies confinement of the original electric photon and disappearance of all the massless modes.
\end{abstract}




\section{Introduction}

The main goal of this paper is to provide a new calculation of the value of the gluino condensate in four-dimensional $\mathcal{N}=1$ supersymmetric pure $S U(N)$ gauge theory. Our approach incorporates recent results and ideas of Refs. [1 7]. Previous to this, two conceptually different approaches for calculating $\left\langle\frac{\operatorname{tr} \lambda^{2}}{16 \pi^{2}}\right\rangle$ have been followed in the literature:

1. In the first methodology [8 10], the so-called strong-coupling instanton (SCI) approach, the gluino condensate $\left\langle\frac{\operatorname{tr} \lambda^{2}}{16 \pi^{2}}\right\rangle$ is determined directly in the strongly coupled theory using an explicit one-instanton calculation of $\left\langle\frac{\operatorname{tr} \lambda^{2}\left(x_{1}\right)}{16 \pi^{2}} \cdots \frac{\operatorname{tr} \lambda^{2}\left(x_{N}\right)}{16 \pi^{2}}\right\rangle$. Cluster decomposition arguments are then invoked in order to extract $\left\langle\frac{\operatorname{tr} \lambda^{2}}{16 \pi^{2}}\right\rangle$.

2. In the second methodology [1], the so-called weak-coupling instanton (WCI) approach, the calculation is performed with additional matter fields whose presence ensures that the theory is weakly coupled and a semi-classical 'constrained instanton' calculation is justified [12]. Holomorphicity of supersymmetric F-terms is then used to decouple the matter fields and to flow to the original pure $\mathcal{N}=1$ gauge theory.

As reviewed in [1], these two methods give two different values for the gluino condensate $10,11,13,14$ :

$$
\begin{aligned}
& \left\langle\frac{\operatorname{tr} \lambda^{2}}{16 \pi^{2}}\right\rangle_{\mathrm{SCI}}=\frac{2}{[(N-1) !(3 N-1)]^{1 / N}} \Lambda^{3} \\
& \left\langle\frac{\operatorname{tr} \lambda^{2}}{16 \pi^{2}}\right\rangle_{\mathrm{WCI}}=\Lambda^{3} .
\end{aligned}
$$

These results are quoted in the Pauli-Villars scheme with $\Lambda$ being the corresponding dimensional transmutation scale of the theory. The reason for the discrepancy between the SCI versus WCI calculations, as well as the question as to which is correct, has been a longstanding controversy [10, 11, 15, 16]. The new ingredient in this old puzzle is the fact that over the last few years multi-instanton technology has been developed [17 21] to the extent that calculations can be performed in supersymmetric (and in principle non-supersymmetric) gauge theories, both in the weak-coupling [17] and in the strong-coupling regimes [21], providing us with successful quantitative tests of, respectively, the Seiberg-Witten solution of $\mathcal{N}=2$ theories [22] and the Maldacena duality [23] in the $\mathcal{N}=4$ theory. In [1] we reexamined the gluino condensate controversy using these recently developed methods. In particular, we evaluated the large $N$ contribution of $k$ instantons to gluino correlation functions and demonstrated conclusively that an essential technical step in the SCI calculation of the gluino condensate, namely the use of cluster decomposition, is actually invalid. 
The central idea pursued in the present paper is that there are additional configurations which contribute to gluino condensate in the strongly-coupled regime, implying that the instanton-induced SCI expression (1.1a) is incomplete. The existence of other contributions necessarily affects the application of cluster decomposition in the following sense. Since each single instanton has $2 N$ adjoint fermion zero-modes the $k$-instanton configuration contributes to the correlation function

$$
\left\langle\frac{\operatorname{tr} \lambda^{2}\left(x_{1}\right)}{16 \pi^{2}} \cdots \frac{\operatorname{tr} \lambda^{2}\left(x_{k N}\right)}{16 \pi^{2}}\right\rangle
$$

rather than directly to $\left\langle\frac{\operatorname{tr} \lambda^{2}}{16 \pi^{2}}\right\rangle$ itself. In the SCI approach gluino condensate is obtained by applying cluster decomposition to (1.2) with the additional assumption that the instanton calculation averages over the $N$ physically equivalent vacua of the $\mathcal{N}=1$ theory. In the following, we will show that the correlator (1.2) is not dominated solely by instantons and hence the clustering argument must be applied to the complete expression and not just to the instanton contribution. This is of course in complete agreement with our earlier observation [1] that clustering fails when only multi-instantons are included in the SCI calculation. Furthermore, when the theory is partially compactified on $\mathbb{R}^{3} \times S^{1}$, we will identify the configurations contributing directly to $\left\langle\frac{\operatorname{tr} \lambda^{2}}{16 \pi^{2}}\right\rangle$ with monopoles. By considering the contribution of the monopole configurations, we will be able to argue that the complete strong coupling expression for gluino condensate is different from the SCI expression (1.1a) but agrees perfectly with the WCI result $(1.1 \mathrm{~b})$.

It has been suspected for a long time [24 27] that in the strongly coupled theories, such as QCD or its supersymmetric brethren, instantons should be thought of as composite states of more basic configurations, loosely referred to as 'instanton partons'. These partons would give important and possibly dominant contributions to the non-perturbative dynamics at strong coupling. Our intention is to make this idea more precise. A necessary evil in our approach is to consider the theory with one of its dimensions compactified.t To this end, we suppose that, say, $x_{4}$, is periodic with period $\beta / 2 \pi$. W We must then impose periodic boundary conditions for bosons and fermions

$$
A_{m}\left(x_{\mu}, x_{4}=0\right)=A_{m}\left(x_{\mu}, x_{4}=\beta\right), \quad \lambda\left(x_{\mu}, x_{4}=0\right)=\lambda\left(x_{\mu}, x_{4}=\beta\right),
$$

\footnotetext{
${ }^{1}$ Our approach is different from the toron calculations of Ref. [28 where all four dimensions were compactified on a torus. The advantage of our method compared to that of [28] is that we do not have to fine-tune the compactification parameters for the finite-action configurations to exist. We also note that the value gluino condensate extracted from the toron approach of [28] in the finite-volume torus with the fine-tuned periods is difficult to interpret in the infinite volume and its numerical value agrees neither with the WCI $1.1 \mathrm{~b})$ nor with the SCI (1.1a) results. In the alternative toron set-up advocated in [29], the fine-tuning problem was avoided at the cost of introducing singular toron-like configurations with a branch cut and an IR regulator.

${ }^{2}$ The indices run over $m=1,2,3,4$ and $\mu=1,2,3$. Our other conventions in four and three non-compact dimensions as well as instanton and monopole basics follow closely Appendices A and C of Ref. [30].
} 
to preserve supersymmetry. An important additional ingredient, as explained in Sec. II of [31, is that the local gauge group itself must also be composed of 'proper' gauge transformations, i.e. those that are periodic on $S^{1}$ :

$$
U\left(x_{\mu}, x_{4}=0\right)=U\left(x_{\mu}, x_{4}=\beta\right)
$$

We will refer to the aforementioned theory as the 'theory on the cylinder $\mathbb{R}^{3} \times S^{1}$ ' to distinguish it from the finite temperature compactification where the fermions have antiperiodic boundary conditions. The situation we envisage is similar to that discussed in [32, since the theory on $\mathbb{R}^{3} \times S^{1}$ interpolates between the four-dimensional $\mathcal{N}=1$ pure gauge theory, for $\beta=\infty$, and a three-dimensional $\mathcal{N}=2$ gauge theory, for $\beta=0$. From now on we will work at finite $\beta$ and only at the end of the calculation take the limit of $\beta \rightarrow \infty$ in order to recover the genuinely four-dimensional theory.

Some time ago, Gross, Pisarski and Yaffe [31] gave a complete topological classification of the smooth finite-action gauge fields on $\mathbb{R}^{3} \times S^{1}$ which may contribute to the path integral in the semi-classical approximation. The relevant configurations are characterized by three sets of invariants: the instanton number $k$, the magnetic charge $q$, and the eigenvalues of the asymptotics of the Wilson line:

$$
\Omega=\mathbb{P} \exp \int_{0}^{\beta} d x_{4} A_{4}\left(x_{4}, x_{\mu} \rightarrow \infty\right) .
$$

One consequence of this classification is that at finite radius $\beta$ instanton configurations do not exhaust the set of semi-classical contributions because configurations with magnetic charges can - and in fact do - contribute to the non-perturbative dynamics including the value of the gluino condensate. Ref. [31] further argued that in the finite temperature compactificationnot the one under present consideration - the non-trivial values of the asymptotic Wilson line (1.5) are suppressed in the infinite volume limit. Consequently, the classically flat directions

$$
\left\langle A_{4}\right\rangle=\operatorname{diag}\left(a_{1}, a_{2}, \ldots, a_{N}\right)
$$

are lifted by thermal quantum corrections and the true vacuum of the theory is $\left\langle A_{4}\right\rangle=0$. In this case the configurations with magnetic charges are not relevant and the semi-classical physics involves instantons only. Remarkably, for the theory on the cylinder, with periodic boundary conditions on the fermions, the argument of [31] does not apply and, as we shall see, the opposite scenario ensues:

(i) The semi-classical physics of the $\mathbb{R}^{3} \times S^{1} S U(N)$ theory is described by configurations of monopoles of $N$ different types. 
(ii) The classical moduli space of the $\mathbb{R}^{3} \times S^{1} S U(N)$ theory (1.6) is lifted in a non-trivial way

$$
\left\langle A_{4}\right\rangle=\operatorname{diag}\left(\frac{N-1}{N} \frac{\pi}{i \beta}, \frac{N-3}{N} \frac{\pi}{i \beta}, \ldots,-\frac{N-1}{N} \frac{\pi}{i \beta}\right),
$$

leaving behind $N$ supersymmetry-preserving vacua labelled by the $N$ discrete values of the $\theta$-angle

$$
\theta_{u}=2 \pi(u-1), \quad u=1,2, \ldots, N
$$

The $N$ vacua are related to each other by the chiral subgroup $\mathbb{Z}_{N}$, which permutes $\theta_{u}$ 's, but leaves the Wilson line (1.7) unchanged. Each such vacuum contributes a factor of 1 to the Witten index $\operatorname{tr}(-1)^{\mathrm{F}}=N$ [33]. The values of gluino condensate in each of these vacua will be related to each other by a trivial phase transformation $\exp \left[i \theta_{u} / N\right]$. From now on we will concentrate on simply one of the vacua, with $\theta_{u}=0$. The distinctive feature of (1.7) is the constant equal spacing between the VEVs $a_{j}$ :

$$
a_{j}-a_{j+1}=\frac{2 \pi}{i N \beta} \bmod \frac{2 \pi}{i \beta}, \quad j=1,2, \ldots, N
$$

In general, one would think that the field configurations of the $\mathbb{R}^{3} \times S^{1}$ theory which are relevant in the semi-classical regime are both instantons and monopoles. Remarkably, however, the instanton configurations are themselves included as specific multi-monopole configurations. This happens in the following way: first of all, an instanton configuration on the cylinder follows from a standard instanton configuration in $\mathbb{R}^{4}$ [34] by imposing periodic boundary conditions in $x_{4}$ (1.3). In addition we need instanton solutions in the presence of a non-vanishing $\mathrm{VEV}$ for the gauge field component $A_{4}$, or equivalently a non-trivial expectation of the Wilson line, as in Eqs. (1.5) and (1.6). Such periodic instantons in the presence of a Wilson line were recently analyzed in Refs. [27].7 It transpires that instantons on the cylinder can be understood as composite configurations of $N$ single monopoles, one of each of the $N$ different types [2], 36, 37]. One expects in an $S U(N)$ theory on $\mathbb{R}^{4}$ that the lowest charged, or fundamental, monopoles come in $N-1$ different varieties, carrying a unit of magnetic charge from each of the $U(1)$ factors of the $U(1)^{N-1}$ gauge group left unbroken by the VEV. The additional monopole, needed to make up the $N$ types, is specific to the compactification on the cylinder [2 5] and will be called here a KK-monpole. The new

\footnotetext{
${ }^{3}$ In general, the $\theta F^{*} F$ term in the microscopic Lagrangian can be rotated away with an anomalous chiral transformation of gluinos. However $\theta$ is an angular variable in the sense that $\theta=2 \pi n, n \in \mathbb{Z}$, is indistinguishable from $\theta=0$. We will demonstrate in the following that the topological charge $Q$ of the configurations contributing to the gluino condensate is $Q=1 / N$ and thus the net effect of the $\theta F{ }^{*} F$ term in each vacuum is the phase factor $\theta_{u} / N$.

${ }^{4}$ For the simpler case of $\left\langle A_{4}\right\rangle=0$ periodic instantons were previously constructed in Refs. [31, 35].
} 
monopole carries specific magnetic charges of the unbroken $U(1)^{N-1}$ gauge group as well as an instanton charge. The magnetic charge of the KK-monopole is such that when all $N$ types of monopoles are present, the magnetic charges cancel and the resulting configuration only carries a unit instanton charge.

The $N-1$ fundamental monopoles are the embeddings of the standard $S U(2)$ BPS monopole 38 41] on $\mathbb{R}^{3}$ spanned by $x_{1,2,3}$ (independent of the $S^{1}$ coordinate $x_{4}$ ) in the gauge group $S U(N)$. At finite radius $\beta$, these monopoles have finite action and hence contribute to the path integral in the semi-classical regime as described in Refs. 442, 43] and [30, 44, 45]. The monopole solutions satisfy Bogomol'nyi equations that are precisely the 4D self-duality equations?

$$
F_{m n}={ }^{*} F_{m n}
$$

and each solution has two adjoint fermion zero-modes as enumerated by the Callias index theorem [46]. The same consideration applies to the KK-monopole as well, since it is at least formally gauge equivalent to a standard fundamental monopole via an improper (nonperiodic) gauge transformation [4]. Since there are two adjoint fermion zero-modes in the background of each of the $N$ types of monopoles, these configurations can contribute directly to $\left\langle\frac{\operatorname{tr} \lambda^{2}}{16 \pi^{2}}\right\rangle$.

The remainder of the paper is organized as follows. Initially, we focus on the case with $S U(2)$ gauge group; the generalization to $S U(N)$ is then obvious. In general, the theory on the cylinder can develop a VEV for the gauge field component along the $S^{1}$ direction, Eq. (1.6), which for the case of $S U(2)$ gauge group we parametrize as

$$
\left\langle A_{4}\right\rangle=v \frac{\tau^{3}}{2 i} \equiv \operatorname{diag}\left(\frac{v}{2 i},-\frac{v}{2 i}\right)
$$

where $v$ is an arbitrary real parameter which parametrizes the classical moduli space. For every fixed $v$ there are actually two distinct vacua corresponding to the choice of theta angle Eq. (1.8). In the Section II, we will show using field theory arguments, backed-up by a D-brane analysis, that:

(i) The classical moduli space is a circle,

$$
v \in S^{1}: \quad 0 \leq v \leq \frac{2 \pi}{\beta} .
$$

\footnotetext{
${ }^{5}$ In the usual interpretation of the self-duality equations for the monopole, the time component of the gauge field is interpreted as the Higgs field; in the present discussion this field is the component of the gauge field along the compact direction.
} 
Consequently, $v$ is an angular variable such that for any fixed $v \neq 0,2 \pi / \beta$, the gauge group is broken to $U(1)$.

(ii) There is a conventional 't Hooft-Polyakov BPS monopole and an additional 'compensating' KK-monopole, each of which satisfies the self-duality equations (1.10) and admits two adjoint fermion zero-modes. The singly-charged instanton solution is a composite configuration of these two monopoles and as expected has four adjoint fermion zero-modes.

Section III is devoted to an evaluation of the monopole-generated superpotential which has the effect of lifting the classical degeneracy parametrized by $v$. We argue that the true quantum vacuum state is simply the point

$$
v_{\mathrm{vac}}=\frac{\pi}{\beta}
$$

Furthermore, at $v=v_{\text {vac }}$ the effective potential is zero and supersymmetry remains unbroken. Hence, there are two supersymmetry-preserving vacua with (1.13) and labelled by $\theta_{1}=0$ and $\theta_{2}=2 \pi$, as per (1.8), in agreement with the calculation of the Witten index $\operatorname{tr}(-1)^{\mathrm{F}}=2[33$. Moreover, we discover that the superpotential not only lifts the classically flat direction, but also gives a mass to the dual (magnetic) photon, which implies confinement of the original electric photon and disappearance of all the massless modes.

Section IV then goes on to consider the monopole contribution to the gluino condensate. In the quantum vacua, the gluino condensate $\left\langle\frac{\operatorname{tr} \lambda^{2}}{16 \pi^{2}}\right\rangle$ receives contributions from both the BPS and KK-monopoles. After summing these contributions, we then take the decompactification limit $\beta=\infty$ to obtain the value of gluino condensate in the strongly coupled $\mathcal{N}=1$ theory which agrees with the WCI calculation (1.1b). Section V concludes with a brief discussion.

\section{Semi-classical Configurations}

In this section, we consider in more detail the configurations which contribute to the semiclassical physics for the theory on the cylinder. We begin with a discussion of the $S U(2)$ case, follow with an alternative description in terms of D-branes and then indicate how the results generalize to the $S U(N)$ gauge group. 


\section{II.1 Gauge group $S U(2)$}

To verify that the classical moduli space is $S^{1}$, consider a non-periodic (hence 'improper') gauge transformation [4]

$$
U_{\text {special }}=\exp \left(\frac{\pi x_{4}}{i \beta} \tau^{3}\right)
$$

Improper gauge transformations are treated differently from proper ones in the path integral, in the sense that two field configurations related by such a gauge transformation do not belong to the same gauge orbit. The transformation $U_{\text {special }}$, however, has a special property: even though it is not itself periodic, $U_{\text {special }}\left(x_{4}=0\right)=-U_{\text {special }}\left(x_{4}=\beta\right)$, the corresponding gauge transformed field configurations:

$$
\begin{aligned}
A_{m}^{\prime} & =\exp \left(\frac{\pi x_{4}}{i \beta} \tau^{3}\right)\left(A_{m}+\partial_{m}\right) \exp \left(-\frac{\pi x_{4}}{i \beta} \tau^{3}\right), \\
\lambda^{\prime} & =\exp \left(\frac{\pi x_{4}}{i \beta} \tau^{3}\right) \lambda \exp \left(-\frac{\pi x_{4}}{i \beta} \tau^{3}\right)
\end{aligned}
$$

remain strictly periodic, i.e.

$$
A_{m}^{\prime}\left(x_{\mu}, x_{4}=0\right)=A_{m}^{\prime}\left(x_{\mu}, x_{4}=\beta\right), \quad \lambda^{\prime}\left(x_{\mu}, x_{4}=0\right)=\lambda^{\prime}\left(x_{\mu}, x_{4}=\beta\right) .
$$

Applied to the third component of the gauge field (1.11), the transformation $U_{\text {special }}$ shifts $v$ according to

$$
\left\langle A_{4}^{\prime}\right\rangle=\left(v-\frac{2 \pi}{\beta}\right) \frac{\tau^{3}}{2 i} .
$$

Thus, using a sequence of these transformation one can ratchet-down an arbitrary value of $v \in \mathbb{R}$, to the range specified in (1.12). In fact the sectors of the theory with $v=\tilde{v}$ and $v=\tilde{v}+2 \pi / \beta$ are physically indistinguishable; one is obtained from the other by relabelling the Kaluza-Klein modes of the compact direction, i.e. relabelling the Matsubara frequencies $\omega_{n}=2 n \pi / \beta$ with $n \in \mathbb{Z}$ associated to the compact $x_{4} \in S^{1}$ variable.

The standard BPS monopole solution in Hedgehog gauge [40,41] is

$$
\begin{aligned}
& A_{4}^{\mathrm{BPS}}\left(x_{\nu}\right)=(v|x| \operatorname{coth}(v|x|)-1) \frac{x_{a}}{|x|^{2}} \frac{\tau^{a}}{2 i}, \\
& A_{\mu}^{\mathrm{BPS}}\left(x_{\nu}\right)=\left(1-\frac{v|x|}{\sinh (v|x|)}\right) \epsilon_{\mu \nu a} \frac{x_{\nu}}{|x|^{2}} \frac{\tau^{a}}{2 i} .
\end{aligned}
$$

These expressions are obviously independent of the $S^{1}$ variable $x_{4}$, since the latter can be thought of as the time coordinate of the static monopole. The boundary values of (2.5) as $|x| \rightarrow \infty$, when gauge rotated to unitary (singular) gauge, agree with (1.11):

$$
A_{4}^{\mathrm{BPS}} \rightarrow v \frac{\tau^{3}}{2 i}=\left\langle A_{4}\right\rangle
$$


The monopole solution (2.5) satisfies the self-duality equations (1.10) and has topological charge

$$
Q \equiv \frac{1}{16 \pi^{2}} \int_{0}^{\beta} d x_{4} \int d^{3} x \operatorname{tr}^{*} F_{m n} F^{m n}=\frac{\beta v}{2 \pi}
$$

There are precisely two adjoint fermion zero modes 46] in the monopole background (2.5). These modes can be generated by supersymmetry transformations of the bosonic monopole components in (2.5), yielding

$$
\lambda_{\alpha}^{\mathrm{BPS}}=\frac{1}{2} \xi_{\beta}\left(\sigma^{m} \bar{\sigma}^{n}\right)_{\alpha}^{\beta} F_{m n}^{\mathrm{BPS}}
$$

Here $\sigma^{m}$ and $\bar{\sigma}^{n}$ are the four Pauli matrices and $\xi_{\beta}$ is the two-component Grassmann collective coordinate; see footnote 2. Finally, the monopole has magnetic charge one, instanton charge zero, and the action $S_{\mathrm{BPS}}$ is

$$
S_{\mathrm{BPS}}=\frac{4 \pi}{g^{2}} \beta v
$$

The monopole of the second type - the KK monopole - can be obtained [幽 from the expressions (2.5) by, firstly, replacing the VEV $v$ on the right-hand side of (2.5) with $2 \pi / \beta-v$, and then gauge transforming the resulting expression with $U_{\text {special }}$ as in (2.2). Finally to install the original $\mathrm{VEV} v$ one performs the reflection $v \rightarrow-v$ implemented by the discrete transformation $U_{\text {refl }}=\exp \left[i \pi \tau^{2} / 4\right]$. The resulting configuration is the KK-monopole $A_{m}^{\mathrm{KK}}$ and, though gauge related to $A_{m}^{\mathrm{BPS}}$, it must, as described earlier, be accounted in the path integral as contributing to a different topological sector.

The improper gauge transformation $U_{\text {special }}$ changes 31 the instanton charge, $k \rightarrow k+q$, and reverses the sign of the magnetic charge, $q \rightarrow-q$. Thus the KK-monopole has instanton charge $k=1$ and monopole charge $q=-1$. The KK-monopole is itself self-dual and its action and topological charge are:

$$
\begin{aligned}
S_{\mathrm{KK}} & =\frac{4 \pi}{g^{2}} \beta\left(\frac{2 \pi}{\beta}-v\right), \\
Q_{\mathrm{KK}} & =1-\frac{\beta v}{2 \pi} .
\end{aligned}
$$

As for the original BPS monopole, there are two adjoint fermion zero-modes (and no antifermion zero-modes) in the KK-monopole background:巳

$$
\lambda_{\alpha}^{\mathrm{KK}}=\frac{1}{2} \xi_{\beta}\left(\sigma^{m} \bar{\sigma}^{n}\right)_{\alpha}^{\beta} F_{m n}^{\mathrm{KK}} .
$$

\footnotetext{
${ }^{6}$ The KK-monopole is self-dual and not anti-self-dual, and the fact that it has negative rather than positive magnetic charge is irrelevant for the fermion zero mode counting.
} 
As was mentioned earlier, Ref. 31] gave a complete topological classification of the smooth finite-action gauge fields on $\mathbb{R}^{3} \times S^{1}$ in terms of the three invariants: the instanton number $k$, magnetic charge $q$ and the VEV $v$, in terms of which

$$
\begin{aligned}
S_{\mathrm{cl}} & =\frac{8 \pi^{2}}{g^{2}}\left(k+q \frac{\beta v}{2 \pi}\right), \\
Q & =k+q \frac{\beta v}{2 \pi} .
\end{aligned}
$$

Comparing Eqs. (2.12) for the BPS monopole and the KK-monopole with the charges of a single instanton, it is tempting to interpret the latter as the mixed BPS-monopole/KKmonopole configuration. This interpretation is made precise in Ref. [3], based on earlier work of Refs. [36, 37] and [2]. We also note that the two gaugino zero-modes of the KKmonopole combined with the two zero-modes of the BPS-monopole produce the requisite four adjoint fermion zero-modes of the $S U(2)$ instanton.

\section{II.2 D-brane description}

Identical conclusions to those reached in Sec. II.1, can be reached in a more geometrical way using D-brane technology and for the additional insight that this point-of-view provides we describe it here.ๆ

For the geometrical interpretation of the construction, the $\mathcal{N}=4$ case is most straightforward; the modification of this set-up relevant to describe the $\mathcal{N}=1$ theory will be considered subsequently. Therefore we begin with two coincident D3-branes whose collective dynamics is described by $\mathcal{N}=4$ supersymmetric $S U(2)$ Yang-Mills on the four-dimensional world volume 48, 49].

We now proceed to wrap the world-volume of our D3-branes on the cylinder $\mathbb{R}^{3} \times S^{1}$, with the radius $R=\beta / 2 \pi$. With this accomplished, one performs a T-duality transformation along the compact direction. The T-duals of the D3-branes are D2-branes stretched along $\mathbb{R}^{3}$ and lying orthogonal to the dual circle $\tilde{S}^{1}$ with radius $\tilde{R}=\alpha^{\prime} / R$. In the presence of the non-trivial Wilson line Eq. (1.11), the D2-branes become separated by a distance $2 \pi \alpha^{\prime} v$ along the direction of the dual circle [49]. Due to the periodicity around the circle, we may restrict $0 \leq 2 \pi \alpha^{\prime} v \leq(2 \pi)^{2} \alpha^{\prime} / \beta$, which is equivalent to Eq. (1.12).

In the T-dual picture, a BPS monopole can be represented by a D0-brane of length $L_{\mathrm{BPS}}=2 \pi \alpha^{\prime} v$ stretched between the two D2-branes. The orientation of the D0-brane (whether

\footnotetext{
${ }^{7}$ For a discussion of $\mathcal{N}=1$ theories and instantons in the context of branes see Ref. [47].
} 
the D0-brane is stretched between the first and second D2-brane, or vice-versa) corresponds to positive or negative magnetic charge, i.e. the monopole or anti-monopole. The monopole mass is the product of the D0-brane tension $\tau_{0}=2 /\left(\alpha^{\prime} g^{2}\right)$ and the D0-brane length $L_{\mathrm{BPS}}$ :

$$
M_{\mathrm{BPS}}=\tau_{0} L_{\mathrm{BPS}}=\frac{4 \pi v}{g^{2}}
$$

in agreement with Eq. (2.9).

Actually, as one might have guessed, there is an infinite tower of monopoles of the same magnetic charge coming from the Kaluza-Klein tower over $\tilde{S}^{1}$ formed by wrapping the D0brane an arbitrary number of times around the circle. Another way to view the same phenomenon, is to consider the freedom to add to a monopole a closed D0-loop starting and ending on the same D2-brane and winding around $\tilde{S}^{1}$; the length of the loop being $L_{\text {loop }}=(2 \pi)^{2} \alpha^{\prime} / \beta$. This D0-loop over the D2-brane can be identified with the instanton. Indeed, after the T-duality transformation along the $\tilde{S}^{1}$ direction, the D2-brane becomes the D3-brane and the D0-loop becomes a D(-1)-brane, or D-instanton. The D3/D $(-1)$ bound-state is the identified with an instanton having charge equal the winding number of the D0-loop over $\tilde{S}^{1}$, and vanishing magnetic charge. The instanton action is in a similar fashion to Eq. (2.13)

$$
S_{\mathrm{inst}}=\beta M_{\mathrm{inst}}=\beta \tau_{0} L_{\mathrm{loop}}=\frac{8 \pi^{2}}{g^{2}},
$$

in agreement with Eq. (2.12). In summary, the standard BPS-monopole is the lowest-lying Kaluza-Klein state with magnetic charge one: the D0-brane between the first and the second D2-brane. The monopole of the second type - the KK-monopole -appears as the D0-brane between the second D2-brane and the first one (hence the magnetic charge $q=-1$ ) completing the dual circle $\tilde{S}^{1}$ (hence carrying instanton number $k=1$ ). Furthermore, the bound-state of the standard and the KK-monopole is the D0-loop, i.e. the instanton. Notice that standard BPS monopole and the KK-monopole (together with their respective anti-monopoles) are the elemental configurations, out of which the whole set of semi-classical configurations with arbitrary $k$ and $q$ can be built.

Although we have described this picture in terms of the $\mathcal{N}=4$ theory, the whole analysis applies to the $\mathcal{N}=1$ case as well with certain modifications. The $\mathcal{N}=1$ four-dimensional Yang-Mills theory is obtained in a D-braney fashion from a configuration of two coincident D4-branes suspended between two NS5-branes, in a manner described in Refs. [50,51. The world-volume of the D4-branes is infinite in four directions $\mathbb{R}^{4}$ and is finite in the fifth direction $\Delta_{5}$, which is the separation between the NS5-branes along the D4-branes. Following the same line of reasoning as in the $\mathcal{N}=4$ case, the infinite part of the world-volume of the D4-branes 
is put on the cylinder and T-dualized. The T-duals of the D4-branes on $\Delta_{5} \times \mathbb{R}^{3} \times S^{1}$ are the D3-branes stretched along $\Delta_{5} \times \mathbb{R}^{3}$ and orthogonal to the dual circle $\tilde{S}^{1}$ with the dual radius $\tilde{R}=\alpha^{\prime} / R$. In the presence of the non-trivial Wilson line Eq. (1.11), the D3-branes become separated 49] by the distance $2 \pi \alpha^{\prime} v$ along the dual circle, which is equivalent to Eq. (1.12).

In the $\mathcal{N}=4$ theory the D2-branes are BPS configurations and consequently, when at rest, there is no interaction between them. Thus, their separation along the dual circle is arbitrary, i.e. $v$ is an arbitrary modulus. In the $\mathcal{N}=1$ theory, the D3-branes are not BPS configurations. In the next Section, via an explicit calculation of a superpotential, we will prove that they actually repel each other. Geometrically this implies that the two D3-branes stay at the opposite ends of the dual circle and consequently $v=\pi / \beta$. Hence, the classically flat direction is lifted precisely in the manner predicted by Eq. (1.13).

The previous set-up described in the context of $S U(2)$ can be immediately generalized to $S U(N)$. We now have $N$ D3-branes positioned along a circle and repelling each other; hence one expects

$$
a_{j}-a_{j+1}=\frac{2 \pi}{i N \beta} \bmod \frac{2 \pi}{i \beta}, \quad j=1,2, \ldots, N
$$

and hence, (1.7). The $N$ types of the monopole-like configurations are composed from $N-1$ standard BPS monopoles represented by the D1-branes of minimal lengths stretched between the adjacent $u^{\text {th }}$ and $(u+1)^{\text {th }}$ D3-branes, $u=1, \ldots, N-1$. The KK-monopole is the D1brane stretched between the $N^{\text {th }}$ and $1^{\text {st }}$ D3-branes. The instanton, as before, is the closed D1-loop around the $\tilde{S}^{1}$ direction.

\section{Evaluation of the Superpotential}

In this section, we will determine the superpotential of the $\mathcal{N}=1$ supersymmetric $S U(2)$ Yang-Mills theory on $\mathbb{R}^{3} \times S^{1}$. The superpotential is trivial in perturbation theory, but receives non-perturbative contributions as described in Ref. 43. Contributions arise from both types of monopole: BPS and KK. As advertised earlier, the classical moduli space (1.12) will be lifted by the superpotential in accordance with Eqs. (1.7) and (1.13).

In the presence of a non-vanishing VEV $v$, fields with isospin components not aligned with the scalar VEV Eq. (1.11), acquire masses $m \propto v$ via the Higgs mechanism. The

massless fields are consequently the $U(1)$ projections $A_{m}^{3}$ and $\lambda^{3}$ of the non-abelian fields $A_{m}^{a}$ and $\lambda^{a}, a=1,2,3$. Moreover, since $x_{4}$ is periodic, each field can be Fourier analyzed as an 
expansion over the discrete Matsubara frequencies $\omega_{n}=2 \pi n / \beta$ with all the $n \neq 0$ modes being massive Kaluza-Klein modes. The $n=0$ modes correspond to the fields independent of the $x_{4}$ coordinate. Thus, the classically massless degrees-of-freedom are the $x_{4}$-independent $U(1)$ fields $A_{\mu}\left(x_{\nu}\right), \phi\left(x_{\nu}\right), \chi\left(x_{\nu}\right)$ and $\tilde{\chi}\left(x_{\nu}\right)$ defined ${ }^{8}$ as in Appendix A of Ref. [30]:

$$
\begin{aligned}
A_{\mu} & =A_{\mu}^{3}: \quad \text { for } \mu=1,2,3 \\
\phi & =A_{4}^{3}, \\
\chi_{\alpha} & =\frac{1}{\sqrt{2}}\left(\lambda_{\alpha}^{3}+\bar{\lambda}_{\dot{\alpha}}^{3}\right), \quad \tilde{\chi}_{\alpha}=-\frac{i}{\sqrt{2}}\left(\lambda_{\alpha}^{3}-\bar{\lambda}_{\dot{\alpha}}^{3}\right) .
\end{aligned}
$$

Here $\chi$ and $\tilde{\chi}$ are the Majorana two-spinors in three-dimensions. The classical action for the massless fields $S_{\mathrm{cl}}^{U(1)}$ can be read-off from the four-dimensional action of $\mathcal{N}=1$ supersymmetric Yang-Mills (cf. [30]):

$$
S_{\mathrm{cl}}^{U(1)}=\frac{\beta}{g^{2}} \int d^{3} x\left(\frac{1}{4} F_{\mu \nu} F^{\mu \nu}+\frac{1}{2} \partial_{\mu} \phi \partial^{\mu} \phi-\frac{1}{2} \chi \hat{\partial} \chi-\frac{1}{2} \tilde{\chi} \hat{\partial} \tilde{\chi}\right),
$$

where $\hat{\partial}=\gamma^{\mu} \partial_{\mu}$, and $\gamma^{\mu}$ are the three-dimensional gamma-matrices. The presence of the monopoles in the microscopic theory means we must also include a surface term $S_{\text {sf }}$ in the action (3.2):

$$
S_{\mathrm{sf}}=-\frac{i \sigma \beta}{8 \pi} \int d^{3} x \epsilon^{\mu \nu \rho} \partial_{\mu} F_{\nu \rho}
$$

Due to Dirac quantization of magnetic charge:

$$
q=\frac{1}{8 \pi} \int d^{3} x \epsilon^{\mu \nu \rho} \partial_{\mu} F_{\nu \rho} \in \mathbb{Z}
$$

in (3.3) $\sigma$ is a periodic Lagrange multiplier variable with period $2 \pi / \beta$.

Following Polyakov [42 an equivalent dual description of the low-energy theory (3.2) and (3.3) can be obtained by promoting $\sigma$ to be a dynamical field $\sigma(x)$. This field serves as the Lagrange multiplier for the Bianchi identity constraint. The classical action for massless fields then contains the terms

$$
\beta \int d^{3} x\left(\frac{1}{4 g^{2}} F_{\mu \nu} F^{\mu \nu}-i \frac{\sigma}{8 \pi} \epsilon^{\mu \nu \rho} \partial_{\mu} F_{\nu \rho}+\cdots\right) .
$$

At this stage the photon field-strength $F_{\mu \nu}(x)$ can be integrated out, and the resulting classical massless action reads

$$
S_{\mathrm{cl}}=\frac{\beta}{g^{2}} \int d^{3} x\left(\frac{1}{2} \partial_{\mu} \gamma \partial^{\mu} \gamma+\frac{1}{2} \partial_{\mu} \phi \partial^{\mu} \phi-\frac{1}{2} \chi \hat{\partial} \chi-\frac{1}{2} \tilde{\chi} \hat{\partial} \tilde{\chi}\right)
$$

\footnotetext{
${ }^{8}$ Note that the calculations in Appendix A of Ref. [30] and the details of the comactification to 3D were given in Minkowski space with $x_{3}$ and not $x_{4}$ being the compactified direction. Here we analytically continue the results of [30] to Euclidean space.
} 
where we have introduced the dual photon scalar field $\gamma(x)$ :

$$
\gamma(x)=\frac{g^{2}}{4 \pi} \sigma(x)
$$

This action is invariant under infinitesimal $\mathcal{N}=2$ supersymmetry transformations in three dimensions:

$$
\begin{aligned}
\delta \phi & =\sqrt{2} \xi_{1}^{\alpha} \chi_{\alpha}-\sqrt{2} \xi_{2}^{\alpha} \tilde{\chi}_{\alpha}, \\
\delta \gamma & =\sqrt{2} \xi_{1}^{\alpha} \tilde{\chi}_{\alpha}+\sqrt{2} \xi_{2}^{\alpha} \chi_{\alpha}, \\
\delta \chi^{\alpha} & =\sqrt{2} \xi_{1}^{\beta} \hat{\partial}_{\beta}{ }^{\alpha} \phi+\sqrt{2} \xi_{2}^{\beta} \hat{\partial}_{\beta}{ }^{\alpha} \gamma, \\
\delta \tilde{\chi}^{\alpha} & =\sqrt{2} \xi_{1}^{\beta} \hat{\partial}_{\beta}{ }^{\alpha} \gamma-\sqrt{2} \xi_{2}^{\beta} \hat{\partial}_{\beta}{ }^{\alpha} \phi
\end{aligned}
$$

It is more convenient for our purposes to use a more compact form of Eqs. (3.6) and (3.8) involving the complex complex scalar $Z$ and fermion $\Psi$ :

$$
\begin{gathered}
Z=\phi+i \gamma, \quad \bar{Z}=\phi-i \gamma \\
\Psi_{\alpha}=\chi_{\alpha}+i \tilde{\chi}_{\alpha}, \quad \bar{\Psi}_{\alpha}=\chi_{\alpha}-i \tilde{\chi}_{\alpha}
\end{gathered}
$$

in terms of which the action is

$$
S_{\mathrm{cl}}=\frac{\beta}{g^{2}} \int d^{3} x\left(\frac{1}{2} \partial_{\mu} \bar{Z} \partial^{\mu} Z-\frac{1}{2} \bar{\Psi} \hat{\partial} \Psi\right)
$$

and the supersymmetry transformations are

$$
\begin{array}{cl}
\delta Z=\sqrt{2} \theta^{\alpha} \Psi_{\alpha}, & \delta \bar{Z}=\sqrt{2} \bar{\theta}^{\alpha} \bar{\Psi}_{\alpha}, \\
\delta \Psi^{\alpha}=\sqrt{2}(\bar{\theta} \hat{\partial})^{\alpha} Z, & \delta \bar{\Psi}^{\alpha}=\sqrt{2}(\theta \hat{\partial})^{\alpha} \bar{Z}
\end{array}
$$

where we have introduced the infinitesimal supersymmetry transformation parameter $\theta^{\alpha}=$ $\xi_{1}^{\alpha}+i \xi_{2}^{\alpha}$.

Non-perturbative quantum effects will modify the classical action for massless fields, Eq. (3.10), by generating a superpotential $\mathcal{W}(\Phi)$ and $\overline{\mathcal{W}}(\bar{\Phi})$ written in terms of the chiral and anti-chiral $\mathcal{N}=1$ superfields:

$$
\begin{aligned}
& \Phi=Z+\sqrt{2} \theta^{\alpha} \Psi_{\alpha}+\theta^{\alpha} \theta_{\alpha} \mathcal{F} \\
& \bar{\Phi}=\bar{Z}+\sqrt{2} \bar{\theta}^{\alpha} \bar{\Psi}_{\alpha}+\bar{\theta}^{\alpha} \bar{\theta}_{\alpha} \overline{\mathcal{F}} .
\end{aligned}
$$

With the addition of this superpotential, the resulting quantum low-energy effective action reads

$$
S_{\mathrm{eff}}=S_{\mathrm{cl}}+\frac{\beta}{g^{2}} \int d^{3} x\left(\int d^{2} \theta \mathcal{W}(\Phi)+\int d^{2} \bar{\theta} \overline{\mathcal{W}}(\bar{\Phi})\right)
$$


As usual, the scalar potential $V_{\text {eff }}$ is determined by the derivatives of the superpotential with respect to the scalar fields

$$
V_{\text {eff }}=\mathcal{F} \overline{\mathcal{F}}=\frac{\partial \mathcal{W}}{\partial Z} \frac{\partial \overline{\mathcal{W}}}{\partial \bar{Z}} .
$$

The true vacuum corresponds to the minimum of $V_{\text {eff }}$. In general $V_{\text {eff }} \geq 0$, and supersymmetry is unbroken only if the vacuum solution has $V_{\text {eff }}=0$.

We are now ready to calculate the superpotential $\mathcal{W}(\Phi)$ and hence the true ground state of the theory in the semi-classical approximation. Since the standard BPS monopole and the KK-monopole have two fermion zero-modes apiece, Eqs. (2.8) and (2.11), they both generate mass terms for classically massless fermions $\bar{\Psi}$, while the corresponding anti-monopoles will generate a mass for $\Psi$ :

$$
\mathcal{L}_{\text {mass }}=\frac{m_{\bar{\Psi}}}{2} \bar{\Psi} \bar{\Psi}+\frac{m_{\Psi}}{2} \Psi \Psi=m_{\bar{\Psi}} \bar{\lambda}^{3} \bar{\lambda}^{3}+m_{\Psi} \lambda^{3} \lambda^{3}
$$

The supersymmetric completion of (3.15) in the low-energy effective action will give the superpotential in question. The masses (3.15) are determined by examining the large distance behaviour of the correlators

$$
\begin{aligned}
G_{\alpha \beta}^{(2)}(x, y) & =\left\langle\lambda_{\alpha}^{3}(x) \lambda_{\beta}^{3}(y)\right\rangle \\
\bar{G}_{\alpha \beta}^{(2)}(x, y) & =\left\langle\bar{\lambda}_{\alpha}^{3}(x) \bar{\lambda}_{\beta}^{3}(y)\right\rangle
\end{aligned}
$$

Using the LSZ reduction formulae, somewhat along the lines of Ref. 443, we find

$$
\begin{aligned}
& G_{\alpha \beta}^{(2)}(x, y) \rightarrow 2 m_{\bar{\Psi}} \beta \int d^{3} X \mathcal{S}_{\mathrm{F}}(x-X)_{\alpha \rho} \epsilon^{\rho \delta} \mathcal{S}_{\mathrm{F}}(y-X)_{\beta \delta} \\
& \bar{G}_{\alpha \beta}^{(2)}(x, y) \rightarrow 2 m_{\Psi} \beta \int d^{3} X \mathcal{S}_{\mathrm{F}}(x-X)_{\alpha \rho} \epsilon^{\rho \delta} \mathcal{S}_{\mathrm{F}}(y-X)_{\beta \delta}
\end{aligned}
$$

Here $\mathcal{S}_{\mathrm{F}}(x)$ is the massless fermion propagator in $3 \mathrm{D}$, or, equivalently, the Weyl-fermion propagator on $\mathbb{R}^{3} \times S^{1}$ with zero Matsubara frequency: $\mathcal{S}_{\mathrm{F}}(x)=\gamma^{\mu} x_{\mu} /(4 \pi|x|)^{2}$.

We first consider the contribution of a single standard BPS-monopole, (2.5) and (2.8), to $m_{\bar{\Psi}}:$

$$
\left\langle\lambda_{\alpha}^{3}(x) \lambda_{\beta}^{3}(y)\right\rangle_{\mathrm{BPS}}=\int d \mu^{\mathrm{BPS}} \lambda_{\alpha}^{\mathrm{LD}}(x) \lambda_{\beta}^{\mathrm{LD}}(y),
$$

where $\lambda_{\alpha}^{\mathrm{LD}}(x)$ is the large distance (LD) limit of the fermion zero modes (2.8) as computed in Appendix C of Ref. [30]:

$$
\lambda_{\alpha}^{\mathrm{LD}}(x)=8 \pi \mathcal{S}_{\mathrm{F}}(x-X)_{\alpha}^{\rho} \xi_{\rho}
$$


and $d \mu^{\mathrm{BPS}}$ is the semiclassical integration measure of the standard single-monopole on $\mathbb{R}^{3} \times S^{1}$ :

$$
\int d \mu^{\mathrm{BPS}}=M_{\mathrm{PV}}^{3} e^{-S_{\mathrm{BPS}}} \int \frac{d^{3} X}{(2 \pi)^{3 / 2}}\left[g^{2} S_{\mathrm{BPS}}\right]^{3 / 2} \int_{0}^{2 \pi} \frac{d \Omega}{\sqrt{2 \pi}}\left[g^{2} S_{\mathrm{BPS}} / v^{2}\right]^{1 / 2} \int d^{2} \xi \frac{1}{2 g^{2} S_{\mathrm{BPS}}} .
$$

This measure is obtained in the standard way by changing variables in the path integral from field fluctuations around the monopole to the monopole's collective coordinates: $X_{\mu}$ (position), $\Omega\left(U(1)\right.$-angle) and $\xi_{\alpha}$ (Grassmann collective coordinates). The relevant Jacobian factors in (3.20) are taken from Ref. 30]. In contradistinction with the 3D calculation of [30, our present calculation is locally four-dimensional, i.e in the path integral we have integrated over the fluctuations around the monopole configuration in $\mathbb{R}^{3} \times S^{1}$. Thus, the UV-regularized determinants over non-zero eigenvalues of the quadratic fluctuation operators cancel between fermions and bosons due to supersymmetry as in Ref. [52].9 The ultra-violet divergences are regularized in the Pauli-Villars scheme, which explains the appearance of the Pauli-Villars mass scale $M_{\mathrm{PV}}$ to a power given by $n_{\mathrm{b}}-n_{\mathrm{f}} / 2=3$, where $n_{\mathrm{b}}=4$ and $n_{\mathrm{f}}=2$ are, respectively, the numbers of bosonic and fermionic zero-modes of the monopole. Collecting together the expressions in Eqs. (2.9), (3.17a), (3.18), (3.19) and (3.20), we find the single-monopole contribution to $m_{\bar{\Psi}}$ is

$$
m_{\bar{\Psi}}^{\mathrm{BPS}}=16 \pi^{2} \beta^{2} M_{\mathrm{PV}}^{3} \exp \left[-\frac{4 \pi}{g^{2}} \beta v\right]
$$

This expression ignores the contributions of monopole-anti-monopole pairs in the background of the single monopole configuration and since monopole-anti-monopole interactions are long-range (Coulombic) their effects are considerable and must be taken into account. This is precisely the famous Polyakov effect [42] and fortunately there is a very elegant way to incorporate it. The interactions of a single monopole with the monopole-anti-monopole medium can be taken into account in a way by simply coupling the monopole to the magnetic photon $\gamma(x)$ (or $\sigma(x)$ ) introduced earlier, in Eqs. (3.3) and (3.7), and at the same time promoting the VEV $v$ to a dynamical scalar field $\phi(x)$. The coupling of the dual photon to the monopole of magnetic charge $q$ is dictated by the surface term in Eq. (3.3). Naturally enough, one is instructed 42, 43] to change the action (2.12) of the original semi-classical configuration as follows:

$$
S_{\mathrm{cl}}=\frac{8 \pi^{2}}{g^{2}}\left(k+q \frac{\beta v}{2 \pi}\right) \rightarrow \frac{8 \pi^{2}}{g^{2}}\left(k+q \frac{\beta \phi(x)}{2 \pi}\right)+i q \beta \sigma(x) .
$$

This means that the mass becomes a local coupling:

$$
m_{\bar{\Psi}}^{\mathrm{BPS}}(x)=16 \pi^{2} \beta^{2} M_{\mathrm{PV}}^{3} \exp \left[-\frac{4 \pi}{g^{2}} \beta \phi(x)+i \frac{4 \pi}{g^{2}} \beta \gamma(x)\right] .
$$

\footnotetext{
${ }^{9}$ In order to invoke the result of [52] one needs the self-duality of the solution, a covariant background gauge, four dimensions and supersymmetry.
} 
It is straightforward to derive the single KK-monopole contribution to $m_{\bar{\Psi}}$. It is obtained in the same way as the expression on the right hand side of (3.21), but instead of $S_{\mathrm{BPS}}$ in (3.20) one has to use $S_{\mathrm{KK}}$ of (2.10): :ण

$$
m_{\bar{\Psi}}^{\mathrm{KK}}=16 \pi^{2} \beta^{2} M_{\mathrm{PV}}^{3} \exp \left[-\frac{4 \pi}{g^{2}}(2 \pi-\beta v)\right] .
$$

The total mass coupling $m_{\bar{\Psi}}(x)$ is then given by the sum of the standard BPS- and the KK-monopole contributions, each embedded into the dual magnetic field theory as per Eq. (3.22):

$$
\begin{aligned}
m_{\bar{\Psi}}(x)= & 16 \pi^{2} \beta^{2} M_{\mathrm{PV}}^{3} \\
& \times\left(\exp \left[-\frac{4 \pi}{g^{2}} \beta \phi(x)+i \frac{4 \pi}{g^{2}} \beta \gamma(x)\right]+\exp \left[-\frac{8 \pi^{2}}{g^{2}}+\frac{4 \pi}{g^{2}} \beta \phi(x)-i \frac{4 \pi}{g^{2}} \beta \gamma(x)\right]\right) .
\end{aligned}
$$

In the second term above, we used the fact that the KK-monopole has $q_{\mathrm{KK}}=-1$ and $k_{\mathrm{KK}}=1$. Denoting the overall coefficient in (3.25) as $M$ :

$$
M \equiv 16 \pi^{2} \beta^{2} M_{\mathrm{PV}}^{3}
$$

and making use of the complex scalar field and fermion of (3.9) we finally get the following expression for the mass term:

$$
\mathcal{L}_{\text {mass }}=\frac{M}{2} \bar{\Psi}(x) \bar{\Psi}(x)\left(\exp \left[-\frac{4 \pi \beta}{g^{2}} \bar{Z}(x)\right]+\exp \left[-\frac{8 \pi^{2}}{g^{2}}+\frac{4 \pi \beta}{g^{2}} \bar{Z}(x)\right]\right) .
$$

This coupling corresponds to a superpotential $\overline{\mathcal{W}}(\bar{\Phi})$ term in (3.13) of the form:

$$
\overline{\mathcal{W}}(\bar{\Phi})=\left(\frac{g^{2}}{4 \pi \beta}\right)^{2} M\left(\exp \left[-\frac{4 \pi \beta}{g^{2}} \bar{\Phi}\right]+\exp \left[-\frac{8 \pi^{2}}{g^{2}}+\frac{4 \pi \beta}{g^{2}} \bar{\Phi}\right]\right) .
$$

Equivalently, the anti-monopoles generate the hermitian conjugate:

$$
\mathcal{W}(\Phi)=\left(\frac{g^{2}}{4 \pi \beta}\right)^{2} M\left(\exp \left[-\frac{4 \pi \beta}{g^{2}} \Phi\right]+\exp \left[-\frac{8 \pi^{2}}{g^{2}}+\frac{4 \pi \beta}{g^{2}} \Phi\right]\right) .
$$

With the expression for the superpotential in hand, we can now calculate the scalar potential (3.14) and determine the true vacuum state of the theory. Consider

$$
\mathcal{F}=\frac{\partial \mathcal{W}}{\partial Z}=-\frac{M g^{2}}{4 \pi \beta}\left(\exp \left[-\frac{4 \pi \beta}{g^{2}} Z\right]-\exp \left[-\frac{8 \pi^{2}}{g^{2}}+\frac{4 \pi \beta}{g^{2}} Z\right]\right) .
$$

\footnotetext{
${ }^{10}$ This is because the KK-monopole is gauge equivalent to the standard monopole with a 'wrong' VEV as explained in the Sec. II.1
} 
The supersymmetry preserving vacuum $\langle Z\rangle=\langle\phi\rangle+i\langle\gamma\rangle$ corresponds to

$$
\mathcal{F}(\langle Z\rangle)=0 \quad \Longrightarrow \quad\langle Z\rangle=\frac{\pi}{\beta},
$$

which corresponds to the scalar VEV $\langle\phi\rangle \equiv v=\pi / \beta$ as predicted in (1.13).

Note that since $\langle\gamma\rangle=0$ the dual photon does not condense, as expected. What is much more interesting is that the dual photon becomes massive,

$$
V_{\mathrm{eff}}\left(\phi=\frac{\pi}{\beta}, \gamma(x)\right)=2\left(\frac{M g^{2}}{4 \pi \beta}\right)^{2} \exp \left[-\frac{8 \pi^{2}}{g^{2}}\right]\left(1-\cos \frac{8 \pi \beta}{g^{2}} \gamma(x)\right),
$$

which implies confinement of the original electric photon and the corresponding disappearance of all the massless modes.

\section{Gluino Condensate from Monopoles}

In this section, we use our description of the quantum vacuum state of the theory to evaluate the monopole contribution to the gluino condensate.

\section{IV.1 Gauge group $S U(2)$}

We are now in a position to directly compute gluino condensate in the $S U(2)$ theory. Firstly, we evaluate the standard BPS monopole contribution to $\left\langle\operatorname{tr} \lambda^{2}\right\rangle$ :

$$
\begin{aligned}
\left\langle\operatorname{tr} \lambda^{2}\right\rangle_{\mathrm{BPS}}=M_{\mathrm{PV}}^{3} e^{-S_{\mathrm{BPS}}} & \int \frac{d^{3} X}{(2 \pi)^{3 / 2}}\left[g^{2} S_{\mathrm{BPS}}\right]^{3 / 2} \int_{0}^{2 \pi} \frac{d \Omega}{\sqrt{2 \pi}}\left[g^{2} S_{\mathrm{BPS}} / v^{2}\right]^{1 / 2} \\
& \times \int d^{2} \xi \frac{1}{2 g^{2} S_{\mathrm{BPS}}} \operatorname{tr}\left(\lambda^{\mathrm{BPS} \alpha}(x) \lambda_{\alpha}^{\mathrm{BPS}}(x)\right),
\end{aligned}
$$

where we have used the expression (3.20) for the monopole measure. To evaluate (4.1), we use the normalization of fermion zero modes from Ref. [30]:

$$
\int d^{3} X \int d^{2} \xi \operatorname{tr}\left(\lambda^{\mathrm{BPS} \alpha}(x) \lambda_{\alpha}^{\mathrm{BPS}}(x)\right)=2 S_{\mathrm{BPS}} \frac{g^{2}}{\beta} .
$$

A straightforward calculation gives

$$
\left\langle\frac{\operatorname{tr} \lambda^{2}}{16 \pi^{2}}\right\rangle_{\mathrm{BPS}}=\frac{1}{2} \frac{\beta v}{\pi} M_{\mathrm{PV}}^{3} \exp \left[-\frac{8 \pi^{2}}{g^{2}} \frac{\beta v}{2 \pi}\right] .
$$


The KK-monopole contribution is obtained by changing $S_{\mathrm{BPS}} \rightarrow S_{\mathrm{KK}}$ in the BPS-expressions above to give

$$
\left\langle\frac{\operatorname{tr} \lambda^{2}}{16 \pi^{2}}\right\rangle_{\mathrm{KK}}=\frac{1}{2}\left(2-\frac{\beta v}{\pi}\right) M_{\mathrm{PV}}^{3} \exp \left[-\frac{8 \pi^{2}}{g^{2}}+\frac{8 \pi^{2}}{g^{2}} \frac{\beta v}{2 \pi}\right] .
$$

The expressions (4.3) and (4.4) explicitly depend on the UV-cutoff $M_{\mathrm{PV}}$ and do not appear to be renormalization group invariant. However, it is pleasing that in the true ground-state established in the last section, this worrisome dependence disappears. At $v=\pi \beta$, we get

$$
\left\langle\frac{\operatorname{tr} \lambda^{2}}{16 \pi^{2}}\right\rangle_{\mathrm{BPS}}=\left\langle\frac{\operatorname{tr} \lambda^{2}}{16 \pi^{2}}\right\rangle_{\mathrm{KK}}=\frac{1}{2} M_{\mathrm{PV}}^{3} \exp \left[-\frac{4 \pi^{2}}{g^{2}}\right] .
$$

Finally, introducing the renormalization group invariant scale $\Lambda_{\mathrm{PV}}$ of the theory via

$$
M_{\mathrm{PV}}^{3} \exp \left[-\frac{4 \pi^{2}}{g^{2}}\right]=\Lambda^{3},
$$

and adding together both monopole contributions we obtain a value for the gluino condensate:

$$
\left\langle\frac{\operatorname{tr} \lambda^{2}}{16 \pi^{2}}\right\rangle=\Lambda^{3}
$$

This is precisely the value obtained in the WCI approach (1.1b).

\section{IV.2 Generalization to $S U(N)$}

The calculation of the superpotential and the gluino condensate can be straightforwardly generalized to the case of $S U(N)$ gauge group. The quantum vacuum has

$$
a_{j}-a_{j+1}=\frac{2 \pi}{i N \beta} \bmod \frac{2 \pi}{i \beta}, \quad j=1,2, \ldots, N
$$

and so each of the $N$ types of monopoles $(N-1$ standard BPS and one KK) have equal actions and equal topological charges:

$$
S_{\text {mono }}=\frac{8 \pi^{2}}{N g^{2}}, \quad Q_{\text {mono }}=\frac{1}{N} .
$$

The contribution of a single monopole to the gluino condensate will be, in analogy with Eq. (4.5),

$$
\left\langle\frac{\operatorname{tr} \lambda^{2}}{16 \pi^{2}}\right\rangle_{\mathrm{BPS}}=\left\langle\frac{\operatorname{tr} \lambda^{2}}{16 \pi^{2}}\right\rangle_{\mathrm{KK}}=\frac{1}{N} M_{\mathrm{PV}}^{3} \exp \left[-\frac{8 \pi^{2}}{N g^{2}}\right] .
$$

The first coefficient of the $\beta$-function is now $b_{0}=3 N$ and the analog of (4.6) reads

$$
M_{\mathrm{PV}}^{3 N} \exp \left[-\frac{8 \pi^{2}}{g^{2}}\right]=\Lambda^{3 N}
$$

Finally, the total contribution of the $N$ monopoles to the gluino condensate, as in $S U(2)$, reproduces the WCI value $(1.1 \mathrm{~b})$. 


\section{Discussion}

More than twenty years ago Polyakov [42] famously observed that in three-dimensional gaugeHiggs theory without fermions the magnetic photon $\gamma(x)$ gets a non-zero mass due to Debye screening in the monopole-anti-monopole plasma. The mass term for the dual photon then implies confinement of the original electric photon. A naïve attempt to generalize this mechanism to four dimensions by simply substituting the three-dimensional instantons (i.e. monopoles) with four-dimensional instantons fails, since in four dimensions instantons and anti-instantons have a dipole-dipole interaction which is short-ranged and hence, the instanton-anti-instanton medium cannot form a Coulomb plasma essential for Polyakov's Debye mechanism. However, it has been suspected for a long time that instantons and antiinstantons can be thought of as composite states of more basic configurations -instanton partons - which would have long-range interactions and lead to a Coulomb plasma and to the Debye screening.

In this paper, following earlier ideas of [8], we have identified the instanton partons with monopoles in the four-dimensional gauge theory compactified on $\mathbb{R}^{3} \times S^{1}$. The Debye screening in the monopole plasma induces a non-zero mass for the dual photon. Hence, we have successfully generalized Polyakov's mechanism of confinement to the four-dimensional supersymmetric gauge theory compactified on $\mathbb{R}^{3} \times S^{1}$.

As the VEVs in Eq. (1.7) are inversely proportional to the radius $\beta$, the theory becomes weakly coupled at small $\beta$ and can be analysed semi-classically. To return to the strongly coupled theory in Minkowski space, we need to consider the opposite limit of large $\beta$. Since all the F-terms are holomorphic functions of the fields and since the VEVs of the fields (1.7) are holomorphic functions of $\beta$, the power of holomorphy [53 allows to analytically continue the semi-classical values of the F-terms to the strong-coupling regime.

As a useful practical application and a test of monopole physics in $\mathbb{R}^{3} \times S^{1}$, we have calculated the value of gluino condensate and taken the decompactification limit to reproduce the WCI result.

We thank Diego Bellisai, Pierre van Baal and Misha Shifman for valuable discussions. VVK and MPM acknowledge a NATO Collaborative Research Grant, TJH and VVK acknowledge the TMR network grant FMRX-CT96-0012 and NMD acknowledges a PPARC studentship. 


\section{References}

[1] T.J. Hollowood, V.V. Khoze, W. Lee and M.P. Mattis, "Breakdown of Cluster Decomposition in Instanton Calculations of the Gaugino Condensate", hep-th/9904116.

[2] K. Lee and P. Yi, Phys. Rev. D56 (1997) 3711, hep-th/9702107.

[3] K. Lee, Phys. Lett. B426 (1988) 323, hep-th/9802012.

[4] K. Lee and C. Lu, Phys. Rev. D58 (1988) 025011, hep-th/9802108.

[5] T.C. Kraan and P. van Baal, Phys. Lett. B428 (1998) 268, hep-th/9802049.

[6] T.C. Kraan and P. van Baal, Nucl. Phys. B533 (1998) 627, hep-th/9805168.

[7] T.C. Kraan and P. van Baal, Phys. Lett. B435 (1998) 389, hep-th/9806034.

[8] V.A. Novikov, M.A. Shifman, A.I. Vainshtein and V.I. Zakharov, Nucl. Phys. B229 (1983) 394; Nucl. Phys. B229 (1983) 407.

[9] G.C. Rossi and G. Veneziano, Phys. Lett. 138B 195; D. Amati, G.C. Rossi and G. Veneziano, Nucl. Phys. B249 (1985) 1.

[10] D. Amati, K. Konishi, Y. Meurice, G. Rossi and G. Veneziano, Phys. Rep. 162 (1988) 169.

[11] V.A. Novikov, M.A. Shifman, A.I. Vainshtein and V.I. Zakharov, Nucl. Phys. B260 (1985) 157.

[12] I. Affleck, M. Dine and N. Seiberg, Nucl. Phys. B241 (1984) 493; Nucl. Phys. B256 (1985) 557.

[13] J. Fuchs and M. G. Schmidt, Z. Phys. C30 (1986) 161.

[14] D. Finnell and P. Pouliot, Nucl. Phys. B453 (1995) 225, hep-th/9503115.

[15] A. Kovner and M. Shifman, Phys. Rev D 56 (1997) 2396, hep-th/9702174.

[16] M. Shifman and A. Vainshtein, "Instantons versus Supersymmetry: Fifteen Years Later", hep-th/9902018.

[17] N. Dorey, V.V. Khoze and M.P. Mattis, Phys. Rev. D54 (1996) 2921, hep-th/9603136; Phys. Rev. D54 (1996) 7832, hep-th/9607202.

[18] N. Dorey, V.V. Khoze and M.P. Mattis, Nucl. Phys. B513 (1998) 681, hep-th/9708036. 
[19] N. Dorey, T.J. Hollowood, V.V. Khoze and M.P. Mattis, Nucl. Phys. B519 (1998) 470, hep-th/9709072.

[20] V.V. Khoze, M.P. Mattis and M.J. Slater, Nucl. Phys. B536 (1998) 69, hep-th/9804009.

[21] N. Dorey, T.J. Hollowood, V.V. Khoze, M.P. Mattis and S. Vandoren, "Multi-Instantons and Maldacena's Conjecture", hep-th/9810243; "Multi-Instanton Calculus and the AdS/CFT Correspondence in $\mathcal{N}=4$ Superconformal Field Theory", Nucl. Phys. B (to appear), hep-th/9901128.

[22] N. Seiberg and E. Witten, Nucl. Phys. B426 (1994) 19, hep-th/9407087.

[23] J. Maldacena, Adv. Theor. Math. Phys. 2 (1998) 231, hep-th/9711200.

[24] A.A. Belavin, V.A. Fateeev, A.S. Schwarz and Y.S. Tyupkin, Phys. Lett. 83B (1979) 317.

[25] V.A. Fateeev, I.V. Frolov and A.S. Schwarz, Nucl. Phys. B154 (1979) 1.

[26] B. Berg and M. Lüscher, Comm. Math. Phys. 69 (1979) 57.

[27] H. Osborn, Ann. Phys. 135 (1981) 373.

[28] E. Cohen and C. Gomez, Phys. Rev. Lett. 52 (1984) 237.

[29] A.R. Zhitnitsky Nucl. Phys. B340 (1990) 56.

[30] N. Dorey, V.V. Khoze, M.P. Mattis, D. Tong and S. Vandoren, Nucl. Phys. B502 (1997) 94, hep-th/9703228.

[31] D.J. Gross, R.D. Pisarski and L.G. Yaffe, Rev. Mod. Phys. 53 (1981) 43.

[32] N. Seiberg and E. Witten, "Gauge Dynamics and Compactification to Three Dimensions", hep-th/9607163.

[33] E. Witten, Nucl. Phys. B202 (1982) 253.

[34] A. A. Belavin, A. M. Polyakov, A. Schwartz and Y. Tyupkin, Phys. Lett. 59B (1975) 85.

[35] B.J. Harrington and H.K. Shepard, Phys. Rev. D17 (1978) 2122.

[36] W. Nahm, "Self-dual Monopoles and Calorons", in: Lecture Notes in Physics 201, Eds. G. Denado et. al. 1984, p. 189.

[37] H. Garland and M.K. Murray, Comm. Math. Phys. 120 (1988) 335. 
[38] G. 't Hooft, Nucl. Phys. B79 (1974) 276.

[39] A.M. Polyakov, JETP Lett. 20 (1974) 194.

[40] E.B. Bogomol'nyi, Sov. J. Phys 24 (1977) 97.

[41] M.K. Prasad and C.M. Sommerfield, Phys. Rev. Lett. 35 (1975) 760.

[42] A.M. Polyakov, Nucl. Phys. B120 (1977) 429.

[43] I. Affleck, J. Harvey and E. Witten, Nucl. Phys. B206 (1982) 413.

[44] J. Polchinski and P. Pouliot, Phys. Rev. D56 (1997) 6601, hep-th/9704029.

[45] N. Dorey, V.V. Khoze and M.P. Mattis, Nucl. Phys. B502 (1997) 94, hep-th/9704197.

[46] C. Callias, Comm. Math. Phys. 62 (1978) 213.

[47] J. Brodie, Nucl. Phys. B532 (1998) 137, hep-th/9803140.

[48] E. Witten, Nucl. Phys. B460 (1996) 335, hep-th/9510135.

[49] J. Polchinski, "TASI Lectures on D-Branes", hep-th/9611050.

[50] S. Elitzur, A. Giveon and D. Kutasov, Phys. Lett. B400 (1997) 269, hep-th/9702014.

[51] E. Witten, Nucl. Phys. B507 (1997) 658, hep-th/9706109.

[52] A. D'Adda and P. Di Vecchia, Phys. Lett. 73B (1978) 162.

[53] N. Seiberg, Phys. Lett. 318B (1993) 469, hep-th/9309335. 